\title{
Evolving Exercise Medicine Concepts growing need for Replicable, Economical \& All Round Exercise Regimens in Developing Countries.
}

\author{
${ }^{1}$ Dr Pavankumar Kohli MBBS MS (orth), DNB(orth) MNAMS (orth), ${ }^{2}$ Dr Sunil \\ Nadkarni MS(Orth) FRCS(Ed) MCh(Orth), ${ }^{3}$ Dr Satishchandra Gore MS(Orth) \\ FABMISS,${ }^{4}$ Bhagyashree Kulkarni MS Microbiology, ${ }^{5}$ Dr Nabh Patil BPTh, \\ MBA- Hospital and Health care \\ ${ }^{I}$ Consultant Sports Orthopedic Surgeon, B.K.L. Walawalkar Hospital, Dervan, India \\ ${ }^{2}$ Consultant Spine Surgeon, B.K.L. Walawalkar Hospital, Dervan, India \\ ${ }^{3}$ Consultant Spine Surgeon, B.K.L. Walawalkar Hospital, Derwan, India \\ ${ }^{4}$ Research Assistant, Department of sports \& orthopedic Surgery, B.K.L. Walawalkar Hospital, Dervan, India \\ ${ }^{5}$ Research Assistant, Department of sports \& orthopedic Surgery, B.K.L. Walawalkar Hospital, Dervan, India
}

\section{Abstract:}

Background: Exercise has become a commercial industry today. The purpose of a healthy mind in a healthy body has become diluted. We tried to answer the question that does extra money and time spent in gyms have extra benefit over traditional holistic exercise program.

Methodology: 20 young healthy adults between 30-40 yrs were divided into 2 groups, 10 who attended the gym and 10 who attended Yoga school to do SuryaNamaskar. At the end of the month, time and money spent on the exercise was evaluated. All participants who before starting exercise were averagely 60-62 points reached 7073 points by $S F-12 \mathrm{~V} 2$ in both systems of training .

Results: Averagely the gym group spent 70 mins six times a week \& Rs 12800 in the first month of training. Comparatively, the Suryanamaskar group spent just 30 mins for six days a week \& had almost no expense. Therefore to achieve same levels of mental and physical fitness, gymgoers needed more than double the time and approximately more than 12 times the expense.

Discussion: The administration should wake up to 1. Need for prevention based health program. 2. Use of traditional systems like yoga to achieve same fitness goals in lesser time with lesser expenses. 3. More studies like this are needed to prevent blind imitation of the western fitness protocols.

Conclusion: Exercises like Surya Namaskar are holistic, need lesser time \& money. In addition such protocols effect simultaneously, endurance, flexibility, strength, core stability, body ergonomics, mind, emotions \& temperament. No separate practices are needed for each of these goals.

Keywords: Suryanamaskar, Yoga, Fitness, time, money, economy, benefits

\section{Introduction}

The need for exercise is well understood in modern world. But whenever the word exercise come to fore, the picture of Gymnasiums, expensive equipment \& personal trainers is the next thought. Thus for the common man, exercise seems unaffordable monetarily. Also in the busy schedule of city life very few people can take out long time from daily responsibilities for exercise and fitness.[1,2] We therefore decided to investigate the most important $\&$ basic question facing potential exercise goers...Are traditional exercise forms less expensive \& time consuming than modern methods of fitness for similar results in average population? Does one have no option to expensive Gymnasiums for ordinary fitness goals?

Does one need hours of complex exercise for basic fitness levels?

\section{Literature Review}

There has been much literature publications for both of types of exercise training, Traditional \& Gymnasium based. They can be broadly classified under following five headings: (photograph 1)

\section{Physical Training}

Sports science, with its progress over the last few years has categorized gains from exercise into three main subsets: A. Endurance (cardiovascular \& muscular) B. Strength, C. Flexibility. These gains of exercise are governed by the all important principle of Specificity i.e. the gains of exercise are particular to the system of training. Thus a marathon runner who focuses on cardiovascular endurance training will be training mainly that particular system and thus will gain mainly in stamina. However he will be unable to match the strength of a weight lifter or the flexibility of a gymnast who focus on training those systems of fitness. To gain on all three 
fronts the athlete must train all the above three modes of physical performance separately and adequately. Although a particular system may be more important for a competitive athlete, none can afford to completely ignore the other faculties of development. Thus a normal adult in the west who is interested in all round physical development, is advised to run for at least $20-30$ mines every day, do weight training at least three times a week and flexibility exercises for $15-20$ minutes every day. Not only does this involve great amounts of time, effort, equipment and expense, the athlete often lags behind in one of the faculties. Sports injuries are the inevitable result. Suryanamaskar is probably the only known mode of exercise that trains all the three faculties of exercise at the same time. Thus $2 / 3^{\text {rd }}$ time is saved to develop the same level of fitness in all three systems and in today's hectic lifestyle that is certainly a huge saving! Also this type of training is a better replication of real life where all faculties are used together rather than individually. In fact the main advantage of Suryanamaskar is that it can be used for any phase of exercise e.g. warm up, main exercise and cool down too.[3-12],

\section{Core Stability}

One of the most important recent principles of sports biomechanics and injury prevention is the concept of Core stability. Specific exercises are required by the sports man for proper core stability. On a biomechanical analysis of Suryanamaskar, it seems that yogis had realized this concept long ago. Proper core stability also helps maintain normal posture and resolve the commonest complaints faced in routine orthopedic practice. $[5,6,13,14]$

\section{Kinetic Chain}

The entire body is kinetic chain .A stiffness or weakness in one area is bound to affect some other part of the body also for example, weak abdominal muscles will often cause an exaggerated curve in the lower spine which often results in backache. Sportsmen who use one side of the body via racquet sports etc often face over development of the dominant side. It simultaneously develops both sides of the body. It is unique in the way that it takes care of the whole kinetic chain from head to toe. $[2,3,5,6,15]$

\section{The Mind}

The greatest value of traditional exercise lies in it not being restricted to the body. It aims to develop Body, mind, emotions and consciousness together. $[5,6,16,17,18,19,20,21]$

\section{Economics}

The LOGIC in favors of Suryanamaskar can be summed up as below:

All the benefits of gaining in all the three modes of regular exercise i.e. endurance building, strength building and Flexibility can be had by doing only one exercise i.e. Suryanamaskar. There is no need for any expensive equipment. Time saving for the same exercise gains and thereafter by stopping energy leakages on negative thoughts and emotions. Suryanamaskar is both diseases preventive and curative. The benefits of mantras, Pranayama and imagery on the Sun are incomparable on the mind, emotions and level of consciousness. No other exercise can give the above mentioned benefits at one go. [5,6,22]

\section{Time}

In todays busy lifestyle time is also a currency. Numerous studies have shown that todays gymgoers waste $30 \%$ of time in non gym activities \& about $50 \%$ dropout within first 3 months. Also time is spent in shopping for sportsgear is much \& gear envy is a known phenomenon.[5,6]

\section{Methods}

A group of 20 young healthy non exercising adults was chosen aged between 30-40 yrs. Their pre exercise SF 12 V2 scores were taken. They were between 60-62 points. This group was further divided into 2 groups, 10 were sent to the Gym \& 10 to Yoga school to do the Yogic circuit exercise Suryanamaskar. At the end of a month , both groups were evaluated on $[24,23]$

1. SF 12 v 2

2. Time spend for the exercise

3. Money spent on training, gear, memberships etc.

\section{Result}

After one month of exercise, both groups reported SF 12 v2 values between $70-73$ points.

There was no major difference between gain in both groups on the short form questionnaire for mental \& physical health.( SF -12 v2) 
However ,On an average, the Gymnasium group group spent Rs 5000 per month on membership \& \& Rs 7800 on gymwear \& kits including shoes. Costs of travel to \& fro to Gyms were not calculated. Average time spent to train all the systems i.e endurance, strength, and flexibility was 60 to 85 mins per day. This included aerobic training for 30 mins thrice a week proceeded \& followed by warm up \& cool down. Strength training was done for large muscle groups \& core was trained thrice a week for 30 mins. Flexibility exercises were done every day for 15 - 20 mins in warm up \& cool down. No injuries were reported in either group. Endurance, Strength \& Flexibility were all given attention as a beginner. Thus averagely a gym goer spent 70 mins six times a week \& Rs 12800 in the first month of training. Comparatively, the Suryanamaskar group spent just 30 mins for six days a week \& had almost no expense. Suryanamaskar served as endurance, strength, flexibility exercise all in one. For gym goers meditative or prayerful state of mind was not trained whereas in Suryanamaskar, it happened automatically with mantras \& prayerful attitude to Sun.

\section{Discussion}

Need for exercise \& its tremendous benefits have been well accepted throughout the scholarly world. The next step is of implementation of exercise i.e which exercise to do \& where. In large populous nations, the issues of money \& time are both important criteria to decide optimum utilization of funds. In a world flooded with information where people do not have the knowledge to take the right decisions, advertisement controls decision. This leads to lots of unnecessary time \& money being spent on superficial gains. The above study has proven that traditional forms of exercise like Yoga can give similar fitness results in half the time \& very little expense. Of course they may not seen as glamorous as well lit \& mirrored gymnasiums but they are more effective. Also the aim of Yoga or traditional exercise is much higher than just pumping iron. It is balanced body,mind \& composed living. Of course for sportspeople with more specific goals, specific programs with weights in gyms etc can be helpful. But one must accept that a summarily useful exercise which can be used as warm up, cool down, main exercise, for gains in strength, flexibility \& endurance at one go viz Suryanamaskar is irreplaceable. And moreover when we add the element of benefits of prayer $\&$ a meditative temperament. $[1,2,3,4,5,19,21]$

The administration should therefore wake up to

\section{Conclusion}

1. Need for prevention based health programs. 2. Use of traditional systems like yoga to achieve same fitness goals in lesser time with lesser expenses. 3. Need for more studies like this to prevent blind imitation of the western fitness protocols.

\section{References}

[1]. Blumenstein, B, Orbach, I, Psychological Skills in Sport : Training and Application. Happauge, NY: Nova Science. Cox, R.H. (1998). Sport Psychology: Concepts and Applications. Boston: McGraw-Hill. 2010

[2]. Warburton DER, Nicol CW, Bredin SSD., Health benefits of physical activity: the evidence. CMAJ., 2006;174:801-9.

[3]. Physical activity and health in Europe: evidence for action edited by Cavill N, Kahlmeier S, Racioppi F, 2006.

[4]. Ross A, Thomas S. The health benefits of yoga and exercise: A review of comparison studies. J Altern Complement Med. 2010;16:3-12.

[5]. Sinha B, Ray US, Sinha TD, Physiological study of Surya Namaskar, a yogic practice, Altern Ther Health Med. 2011;17(3):62-3.

[6]. Omkar SN, Surya Namaskaar for Holistic Well Being: A Comprehensive Review of Surya Namaskaar, Groessl, J Yoga Phys Ther 2012, 2:2

[7]. Physical Activity and Cardiovascular Disease Prevention in the European Union 1999. http://www.ehnheart.org/aboutus/overview.html

[8]. Sinha B, Ray US, Pathak A, Selvamurthy W, Energy cost and cardiorespiratory changes during the practice of Surya Namaskar, Indian J Physiol Pharmacol. 2004; 48(2):184-90.

[9]. Bhutkar PM, Bhutkar MV, Taware GB, Doijad V, Doddamani BR, Effect of Suryanamaskar Practice on Cardio-respiratory Fitness Parameters: A Pilot Study. Al Ame en J Med Sci. 2008; 1(2): 126-129.

[10]. Mody BS, Acute effects of Surya Namaskar on the cardiovascular \& metabolic system, J Bodyw Mov Ther. 2011;15(3):343-7.

[11]. Karthik PS, Chandrasekhar M, Ambareesha K, Nikhil C, Effect of pranayama and suryanamaskar on pulmonary functions in medical students, J Clin Diagn Res. 2014 Dec;8(12):BC04-6.

[12]. Sinha B, Sinha TD, Effect of 11 months of yoga training on cardiorespiratory responses during the actual practice of Surya Namaskar,Int J Yoga. 2014; 7:72-75.

[13]. Godse AS, Shejwal BR, Godse AA, Effects of suryanamaskar on relaxation among college students with high stress in Pune, India, Int J Yoga. 2015; 8(1): 15-21

[14]. Coe DP, Pivarnik JM, Womack CJ, Reeves MJ, Malina RM. Effect of physical education and activity levels on academic achievement in children, Med Sci Sports Exerc. 2006 Aug;38(8):1515-9.

[15]. Sibley, BA, Etiner, JL, The relationship between physical actively and cognition in children: a meta-analysis. Pediatric Exercise Science, 2003; 15, 243-256.

[16]. Larun L, Nordheim LV, Ekeland E, Hagen KB, Heian F. Cochrane Database of Systematic Reviews. Cochrane AN: 2009. Exercise in prevention and treatment of anxiety and depression among children and young people; p. CD004691.

[17]. Page RM, Hammermeister J, Scanlan A, Gilbert L. Is school sports participation a protective factor against adolescent health risk behaviors? J Health Educ 1998;29:186-192. 
[18]. Pyle RP, McQuivey RW, Brassington GS, Steiner H. High school student athletes: associations between intensity of participation and health factors, Clin Pediatr (Phila). 2003;42(8):697-701..

[19]. Scully D, Kremer J, Meade MM, Graham R, Dudgeon K, Physical exercise and psychological well being: a critical review, Br J Sports Med 1998;32:111-120

[20]. Newsom TJ, Shaw BA, August KJ, Strath SJ, Physical activity-related social control and social support in older adults: Cognitive and emotional pathways to physical activity, JHP. 2016; 1-7

[21]. Kok BE, Coffey KA, Cohn MA, Catalino LI, Vacharkulksemsuk T, Algoe1 SB, Brantley M, Fredrickson BL, How Positive Emotions Build Physical Health: Perceived Positive Social Connections Account for the Upward Spiral Between Positive Emotions and Vagal Tone, APS., 24(7): 1123-1132.

[22]. Pratt M, Macera CA, Wang G, Higher Direct Medical Costs Associated With Physical Inactivity, The Physician and Sports medicine , 2015; 63-70

[23]. Clark CJ, Problem Solving and Personality Factors of Two At-risk College Populations. Unpublished doctoral dissertation, University of West Virginia, 2002.

[24]. Schofield MJ, Mishra G, Validity of the SF-12 Compared with the SF-36 Health Survey in Pilot Studies of the Australian Longitudinal Study on Women's Health, Journal of Health Psychology, 1998; 3(2):259-27.

[25]. Vinga SD, Malmendier V, Paying Not to Go to the Gym, 2006; 96(3): 694-719.

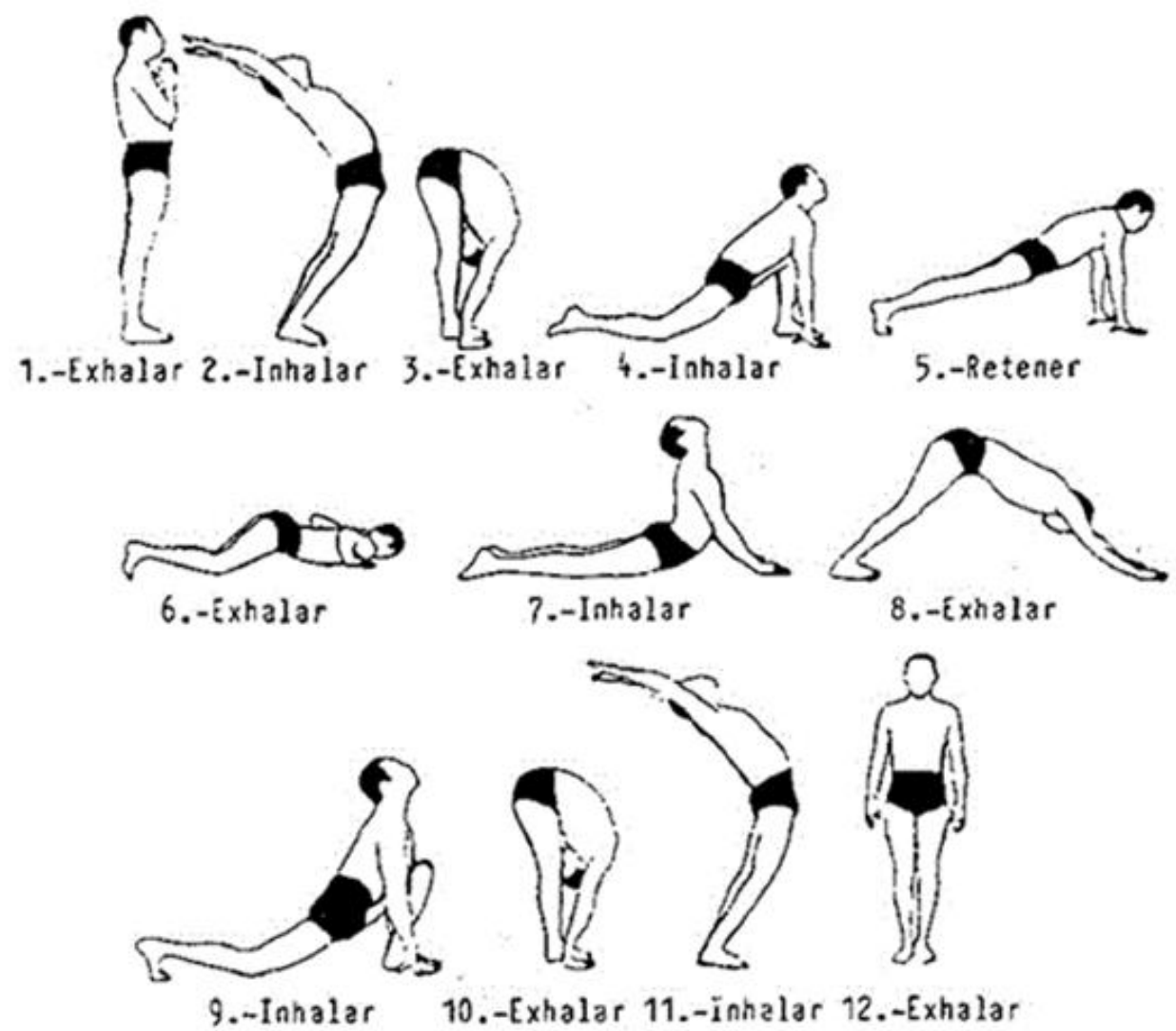

Figure 1: Steps of Suryanamaskar with Breathing Protocol 